\title{
On-machine measurement and error analysis for narrow neck thickness based on ultra-precision machine tool
}

Xin Jin

Beijing Institute of Technology

Ruilin Gao

Beijing Institute of Technology

Chaojiang Li ( $\nabla$ mecjli@bit.edu.cn )

Beijing Institute of Technology

Zhongpeng Zheng

Beijing Institute of Technology

Muzheng Xiao

Beijing Institute of Technology

Zhen Zuo

Beijing Institute of Technology

\section{Research Article}

Keywords: Ultra-precision machining, On-machine measurement, Contact inductive probe, Narrow neck thickness, Error analysis

Posted Date: March 10th, 2021

DOl: https://doi.org/10.21203/rs.3.rs-299877/v1

License: (9) This work is licensed under a Creative Commons Attribution 4.0 International License. Read Full License 


\section{On-machine measurement and error analysis for narrow neck thickness based on ultra-precision machine tool}

Xin Jin ${ }^{1}$, Ruilin Gao ${ }^{1}$, Chaojiang Li ${ }^{*}$, Zhongpeng Zheng ${ }^{1}$, Muzheng Xiao ${ }^{1}$, Zhen Zuo ${ }^{{ }^{*}}$

1) Key laboratory of Micro Manufacturing Technology, School of mechanical and engineering, Beijing Institute of Technology, Beijing 100081, China

* Corresponding authors : Chaojiang Li: mecjli@ bit.edu.cn; ORCID: 0000-0002-5193-6779

Zhen Zuo:zhen_zuo@163.com

Abstract: As flexible joint is a typical low-rigidity micro part, which have four narrow neck structures evenly distributed around its central axis, it is necessary to strictly control the dimensional accuracy of the narrow necks to improve the consistency of stiffness. However, the radius of the arc of the narrow necks is less than $2 \mathrm{~mm}$, and the thickness of thinnest part of the necks formed by two adjacent arcs is only tens of microns, which also has sub-micron accuracy requirement. These cross-scale dimensional characteristics and accuracy requirements give rise to extremely difficulty on the measuring process. In this paper, an on-machine measurement method for the semicircular narrow necks was presented and a measuring device was developed based on the comparative principle by making full use of the high linearity characteristic in the small measuring range of the sensor probes. Meanwhile, the on-machine measurement process based on ultra-precision machine tools was also introduced in details. The experiments results show that the measurement error of the method proposed is less than $0.2 \mu \mathrm{m}$, and the repeatability is less than $0.1 \mu \mathrm{m}$, which meet the measuring requirement of flexible joint. Furthermore, the theoretical error and the uncertainty caused by probe position, measurement force, environmental factor and the accuracy of the sensor was analyzed, which could provide further evidences to improve the measuring accuracy of micro-scale hybrid surface texture.

Keywords: Ultra-precision machining, On-machine measurement, Contact inductive probe, Narrow neck thickness, Error analysis

\section{Introduction}


With the development of high precision, light weight and miniaturization, mechanical parts that has the structural characteristics of miniature, light weight, hybrid surface texture and basic characteristics of low rigidity and high precision, have been widely used in aerospace, medical, defense, communications and electronics industries ${ }^{[1][2]}$. For example, the flexible joint in the inertial device shown in Fig.1, has four circular narrow neck structures evenly distributed around the central axis, and each pair of narrow neck structures can produce bending stiffness along a certain direction. The dimensional accuracy of the narrow necks is important to improve the stiffness consistency, which determines the performance of the parts. However, there is a cross-scale dimensional characteristic. For example, the radius of semicircular arc is around $2 \mathrm{~mm}$, and the thickness of thinnest part formed by two arcs is only tens of microns, while the accuracy of narrow necks is required to be sub-micron scale, which add the measurement difficulty in terms of high accuracy and limited space. The existing processing technology usually adopts off-line methods. i.e the stiffness is tested after the parts are processed, and then the necks are processed again according to the test results. The repeated processing and testing operation not only make the processing cycle longer, but may cause secondary clamping error as well ${ }^{[3]}$, which lead a low product qualification rate. Therefore, the on-machine measuring method of the narrow neck thickness is needed to solve the problems above to ensure the machining accuracy and functional requirements of the parts ${ }^{[4]}$.

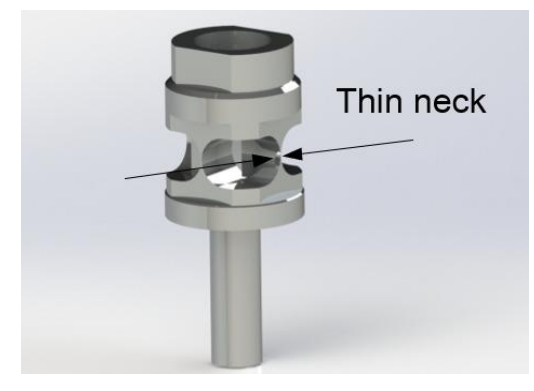

Fig. 1 The flexible joint

For thickness measurement and sub-micron size measurement methods, J. Zhao ${ }^{[5]}$ developed a high-precision digital image correlation method for measuring the radial run-out of rotation by using a low-cost dial indicator and a portable USB digital microscope, and the accuracy can reach sub-micron level. M. Lian ${ }^{[6]}$ proposed an ultrasonic automatic scanning technology for the thickness measurement of large 
narrow-walled parts. The position of the ultrasonic sensor can be adjusted adaptively according to the surface contour of the parts, and the measurement accuracy can be controlled within $0.04 \mathrm{~mm}$. Shahabi et al. ${ }^{[7]}$ used machine vision technology for roughness evaluation and proposed a method of extracting the two-dimensional contour from the edge image of the workpiece for roughness measurement, and results showed that the error is within $10 \%$ when comparing with the traditional measurement using contact probe. Böhm $\mathrm{J}$ et al. ${ }^{[8]}$ used the angle-resolved light scattering method to detect scratches on high-gloss metal surfaces. The shallowest scratches this system can detect are $1.40 \mathrm{~nm}$ wide and $40 \mathrm{~nm}$ deep. In addition, there are also optical microscope method, capacitance method, laser interferometry method, confocal detection method and so on ${ }^{[9]}$ for the measurement in sub-micron scale.

On-line and on-machine measuring process is widely used for its rapidity: G. Yan et al ${ }^{[10]}$ proposed a novel approach of three-linear-axis ultra-precision grinding with wheel path generation, tool interference checking and profile compensation. The new wheel path is generated based on the previous wheel path and the measured profile error.And the result showed the profile accuracy can achieve $100 \mathrm{~nm}$ in peak to valley with surface roughness less than $8 \mathrm{~nm}$ in Sa.Suzuki et al ${ }^{[11]}$ developed an on-line surface measurement method to measure aspherical parts on an ultra-precision grinder by using a contact probe. The probe maintains the contact angle with the aspherical surfaces during the measurement process to reduce the change of the probe friction force, this measurement method can achieve a resolution of $0.14 n m$. Chen et al ${ }^{[12]}$. developed an on-line contact method to detect the surface profile and applied it to ultra-precision mold grinding, then used the results of on-line inspection to establish a new tool path and corrected errors along the surfaces. The final compensation grinding process achieved a profile accuracy PV of $177 \mathrm{~nm}$ and a surface roughness $R a$ of $1.7 \mathrm{~nm}$. Zhang et al. ${ }^{[13]}$ combined on-line and off-line measurement results and improved machining accuracy of diamond freeform surfaces. Noh et al. ${ }^{[14]}$ and Lee et al. ${ }^{[15]}$ innovatively integrated the piezoelectric force sensor into the fast tool servo (FTS) device, which can be used as tool and force sensor after processing. Li et al. ${ }^{[16]}$ integrated an interferometer on the ultra-precision lathe, under the premise of maintaining the consistency of the processing coordinate system, the surface error 
obtained from on-line measurement result is directly fed back to the correction operation by modifying the tool trajectory, which realized nano-scale resolution. In order to overcome the strict environmental requirements of machine optical measurement systems, Li et al. ${ }^{[1]}$ proposed a three-dimensionalon on-machine measurement system based on the principle of disparity pattern-based autostereoscopic(DPA), which is used for measuring micro-structured surfaces on ultra-precision machine tools. The system used a microlens array to capture the original three-dimensional information to establish a three-dimensional mathematical model of the target surfaces, and extracted parallax information to achieve three-dimensional reconstruction of complex surfaces in different measurement environments. Based on statistical data, sub-micron measurement repeatability was achieved through an error elimination process.

Although the research mentioned above has achieved sub-micron or even nanometer-level precision measurement, the measured object usually focus on the characteristic of single surface ${ }^{[18-19]}$. Few measurement methods are suitable for the thickness of complex microstructures of microparts. As for the narrow necks of flexible joint mentioned in this paper, the structures under measurement are complex, and there is a large scale difference between the neck size and the accuracy requirements. For non-contact measurement method, such as microscopic image method, the improvement of measurement accuracy will place higher requirements on the hardware of the entire system, leading to a higher cost ${ }^{[20]}$. And the measurement method based on optical principle is vulnerable to the environmental brightness and

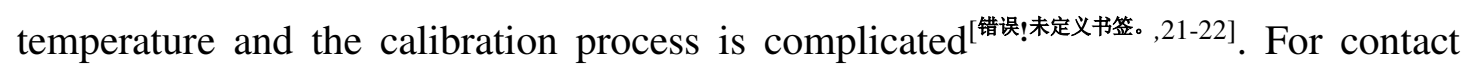
measurement method, the touch trigger usually runs at a lower scanning speed, and the presence of contact force makes them unsuitable for measuring super smooth and soft surfaces, leading to a low measuring efficiency ${ }^{[23]}$. At the same time, sensors suitable for contact measurement methods such as inductive sensors will have different linearity in different ranges, and the instability of the measurement process and the manufacturing errors of the fixtures will also affect the measurement accuracy. And the limitation of the measurement space is a common problem for both contact measurement and non-contact measurement. 
In order to solve the problems such as limited measurement space, scale difference between the objects and accuracy requirements, sensitivity to environmental change, low measurement accuracy and efficiency, this paper proposes a narrow neck on-machine measurement method based on the comparative principle. Then the theoretical measurement error and the uncertainty caused by factors such as motion errors, environmental change and so on has been analyzed, the experiment results show that the method realizes high efficiency and high accuracy cross-scale measurement of the narrow neck structures.

\section{Measuring method}

\subsection{Sensor selection and measuring device}

GT22HP inductive probe and TT80 display unit from TESA company are chosen and the performance of which are shown in Table 1.

Table 1 :The performance of the sensor and display unit

\begin{tabular}{cccccc}
\hline Linearity & Repeatability & Measuring & Measuring principle & Range & Resolution \\
& force & & \\
$0.07+0.4$ & $0.01 \mu m$ & $0.63 N$ & Comparative & $\pm 0.5 \mu m- \pm 5000 \mu m$ & $0.01 \mu m$ \\
$L(\mu \mathrm{m})$ & & & measuring method \\
\end{tabular}

$L$ is the measuring lentgh of the object in $\mathrm{mm}$.

The measuring principle is based on the comparative measurement method: the probe A and B can calculate the measurement length according to different calculation modes such as $A+B, A-B,-A+B,-A-B$, so we can choose a workpiece as the standard, and then perform comparison operations. In this study, we use a standard gauge as a standard to zero the sensor, and then narrow neck is intervened between the two probes, and the thickness difference between which and the gauge is then calculated(i. e. $\sigma_{\mathrm{A}}+\sigma_{\mathrm{B}}$, the total compression of $\mathrm{A}$ and B), as shown in Fig.2a, This can make full use of the small range linearity of the sensor, thereby further improve the measurement accuracy. In addition, a appropriate stylus has been customized to meet the space requirements when measuring, and the measuring device has been developed according to the measuring principle, which is shown in Fig.2b. 

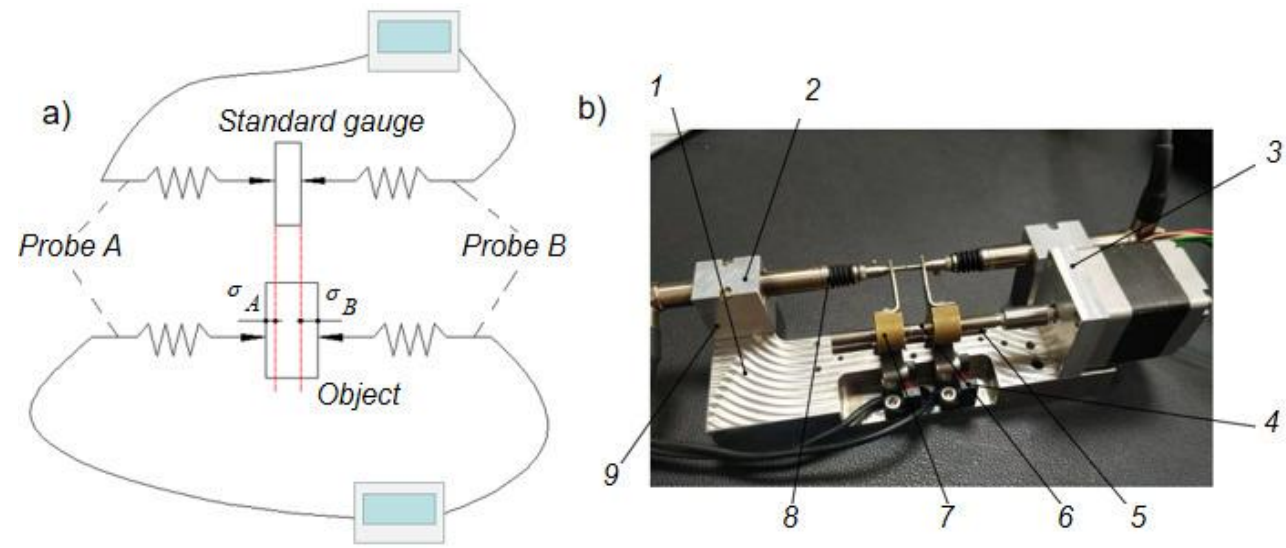

Fig. 2 Measuring method. a) comparative measurement principle. b) measuring device.

(1-base, 2-V-shape cover, 3-stepper motor, 4-photoelectric switch, 5-rail component, 6-screw, 7-fork assemblies, 8-inductive probe, 9-V-shape block)

The carrier for the on-line measurement of the narrow necks of flexible joints is our ultra-precision turn-milling compound machine tool, which has three macro axes $\mathrm{X}, \mathrm{Y}, \mathrm{Z}$, two rotation axes $\mathrm{B}$ and $\mathrm{C}$ and two micro axes $\mathrm{U}$ and $\mathrm{W}$. The machine coordinate system is shown in Fig.3a. The relative position between the measuring device and the machine tool is shown in Fig.3b.

In this paper, the flexible joint is clamped on the spindle of the machine tool, and the neck is finally grounded to meet the technical requirements. If the thickness measured is too large, the grinding process will continue until the thickness is qualified.

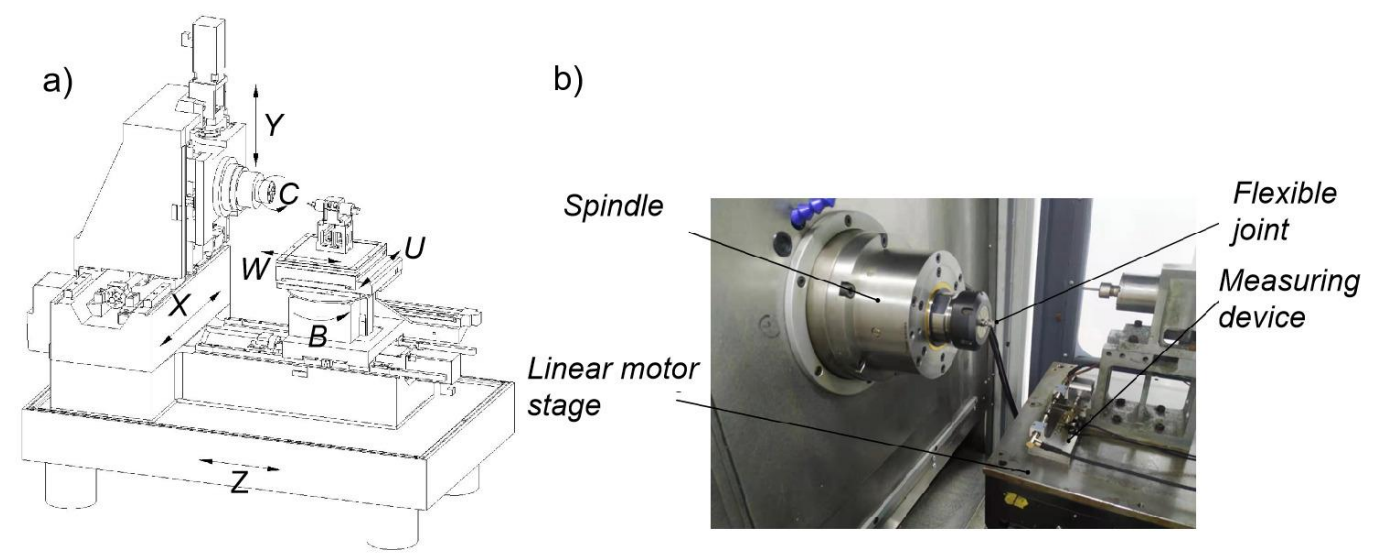

Fig.3 The carrier for the on-machine measurement. a) Machine coordinate system. b) Position of the measuring device

\subsection{Calibration}

Before the experiment, the sensors need to be calibrated first ${ }^{[24]}$. The 
measurement of the narrow neck thickness is a static measurement, so the measuring instrument is calibrated under static standard conditions. The environmental temperature is $20.1{ }^{\circ} \mathrm{C}$ and the relative humidity is $65 \%$. The values of standard gauge blocks is from 10 to $40 \mu m$ and 70 to $90 \mu m$. A digital display horizontal Length measuring instrument is used as the calibration standard, which is shown in Fig.4, and the values measured by which are set as theoretical value inputs, while the measurement values of the measuring device are set as actual value outputs.

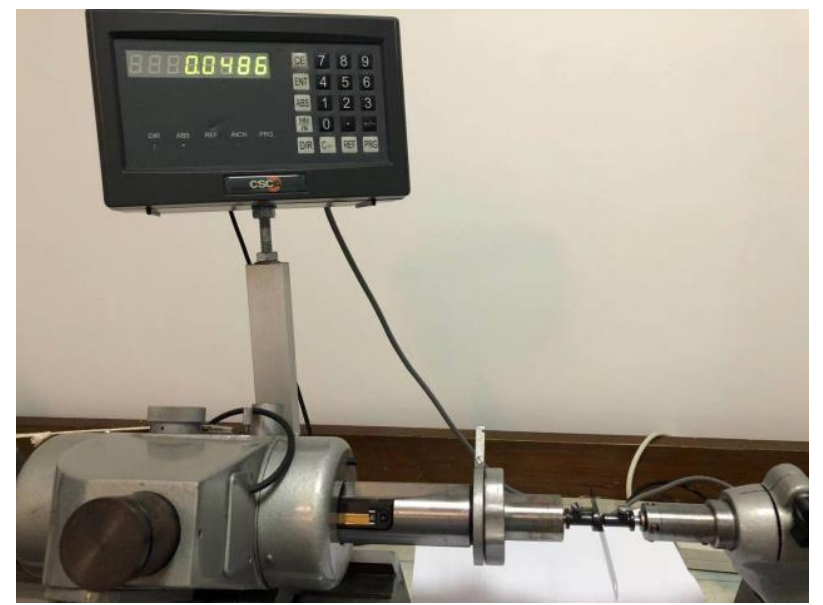

Fig.4 A digital display horizontal Length measuring instrument

After the calibration test of the sensors. We obtained 7 sets of data, the calibration data is shown in Table 2.

Table 2 Calibration data of measuring device: unit $(\mu m)$

\begin{tabular}{cccccc}
\hline Gauge size $(\mu m)$ & \multicolumn{3}{c}{ Measuring value $(\mu m)$} & average $(X / \mu m)$ & indication $(Y / \mu m)$ \\
\hline 10 & 10.34 & 10.39 & 10.34 & 11.62 & 10.60 \\
20 & 19.65 & 19.72 & 19.65 & 22.36 & 19.90 \\
30 & 30.30 & 30.20 & 30.28 & 33.22 & 30.27 \\
40 & 40.72 & 41.00 & 40.90 & 39.44 & 40.93 \\
70 & 70.78 & 70.80 & 70.90 & 70.40 & 70.87 \\
80 & 80.08 & 79.79 & 80.07 & 83.42 & 79.90 \\
90 & 90.47 & 90.50 & 90.49 & 91.22 & 90.46 \\
\hline
\end{tabular}

We used least squares method to fit a straight line, which is shown in Fig.5. The fitted linear equation is $\mathrm{Y}=0.9998 \mathrm{X}+0.0151$, The linearity of the measuring device is $0.256 \%$. 


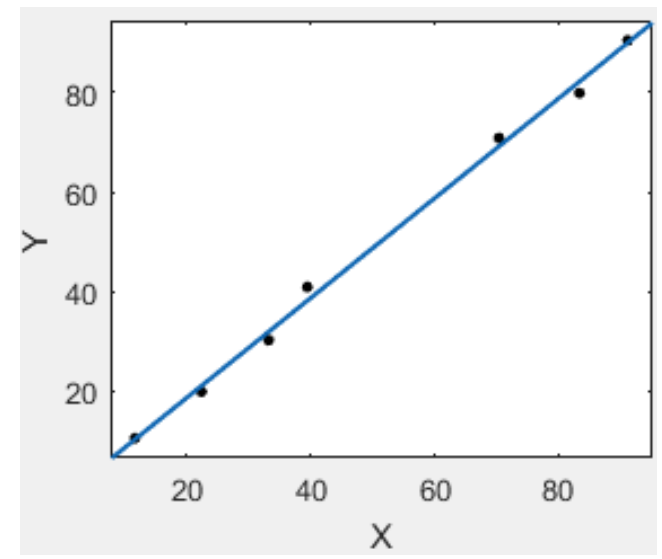

Fig.5 Fitting line

\subsection{Measurement procedure}

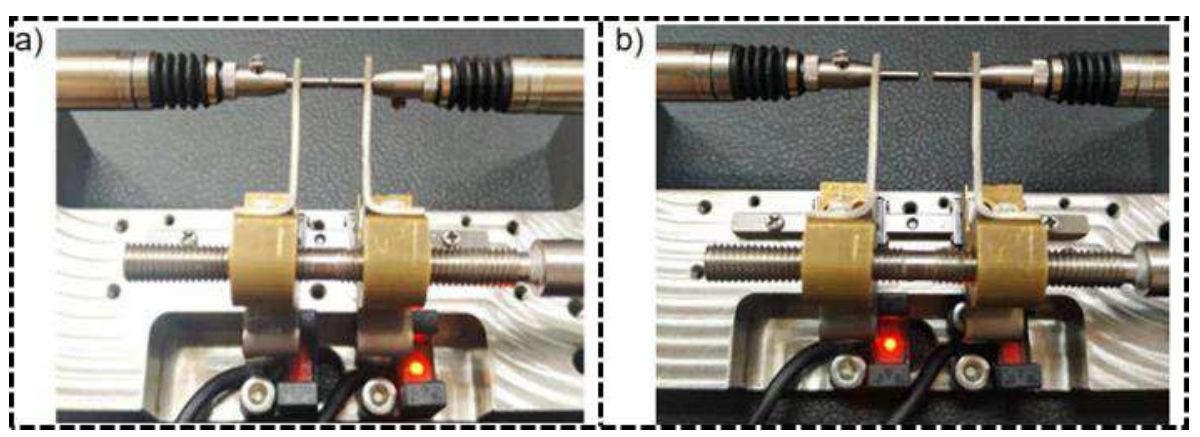

Fig.6 Motion of the probes. a) Two probes contact with each other. b) Two probes move away

After the calibration, the measuring device is installed on the Linear motor stage in $\mathrm{W}$-direction of the machine tool. The on-machine measurement process is as follows:

Step 1:Before the measurement, we assume that the two probes contact with each other, as shown in Fig.6a. The stepper motor then drives the screw and the shift fork moves the front end of the inductive probe away until the photoelectric switch stops the motor, which is shown in Fig.6b.

Step 2:The workpiece on the spindle moves to the specified position along the $\mathrm{X}$ and $\mathrm{Y}$ axes, and the measuring device approaches the workpiece along the $\mathrm{Z}$ axis until the then neck inserts two open probes, and then the stepper motor rotates in the opposite direction. The front end of the inductive probe stretches back and clamps the neck of the flexible joint. Then the motor stops and the precision sliding stage in $\mathrm{W}$ direction moves back and forth, finally we get a minimum value in the display unit and the neck size can be calculated.

Step 3:The motor drives the screw and the fork pushes the front end of the probe 
away, and then flexible joint moves out of the measuring device.

\subsection{Experiment setup}

After completing the calibration and performance inspection of the measuring device, the measurement experiment of the flexible joints can be carried out according to the measurement procedure in Sect.2.3. For the existing flexible joint samples, we obtained 10 sets of measuring data. By corresponding to the calibrated fitting straight line, the residual error between the theoretical values and the actual values of the narrow necks was obtained, the result is discussed in Sect.3.1.

\section{Results and discussion}

\subsection{Measuring results of flexible joint samples}

We regard the residual error as a random distribution, then calculate the standard deviation $\sigma$, and use $3 \sigma$ as the measurement error. The range of measurement data of each group is regarded as the repeatability error. The experimental data of measurement is shown in Table 3.

Table 3 Measurement data of narrow neck thickness: unit $(\mu m)$

\begin{tabular}{ccccccccccccccc}
\hline No. & $B 1$ & $B 2$ & \multirow{2}{*}{$B 3$} & $B 4$ & $B 5$ & $B 6$ & $B 7$ & $B 8$ & $B 9$ & $B 10$ & $\bar{B}$ & $\Delta R_{i}$ & $3 \sigma$ \\
\hline 1 & 57.29 & 57.28 & 57.33 & 57.34 & 57.37 & 57.37 & 57.28 & 57.30 & 57.35 & 57.30 & 57.32 & 0.09 & 0.11 \\
2 & 56.25 & 56.26 & 56.24 & 56.30 & 56.25 & 56.28 & 56.34 & 56.28 & 56.23 & 56.28 & 56.27 & 0.11 & 0.10 \\
3 & 53.30 & 53.23 & 53.24 & 53.22 & 53.22 & 53.34 & 53.32 & 53.30 & 53.20 & 53.22 & 53.26 & 0.14 & 0.15 \\
4 & 54.66 & 54.68 & 54.74 & 54.75 & 54.67 & 54.65 & 54.66 & 54.70 & 54.66 & 54.70 & 54.69 & 0.10 & 0.11 \\
\hline
\end{tabular}

It can be seen from Table 3 that the measurement error of the device is less than $0.15 \mu \mathrm{m}$, and the repeatability error is less than $0.1 \mu \mathrm{m}$, which satisfies the measuring requirement of flexible joint.

\subsection{Analysis of different measurement error sources}

We believe that the manufacturing error of the measuring device, the movement error of the machine tool, the deformation of the workpiece caused by the measuring force, the environmental factor(temperature only) and the characteristics of the sensor itself will all affect the measurement result ${ }^{[25]}$. Therefore, the significance analysis of each error source has been carried out to provide further evidences to optimize the measurement accuracy. 


\section{(1)The measurement error due to probe position:}

Due to the movement error of the micro-motion stage in $\mathrm{W}$ direction, the probe will be eccentric. The measurement error diagram is shown in Fig.7a. From the geometric relationship, the measurement error can be formulated as Equation(1).

$$
\Delta L=L-0.04=2 R-2\left(r+\sqrt{(R-r)^{2}-h^{2}}\right)
$$

Where $r$ is probe radius, $R$ is arc radius, $h$ is the offset from the center in $\mathrm{Z}$ direction. $L$ is the actually measured thickness, $\Delta L$ is the difference between ideal thickness and $L$.

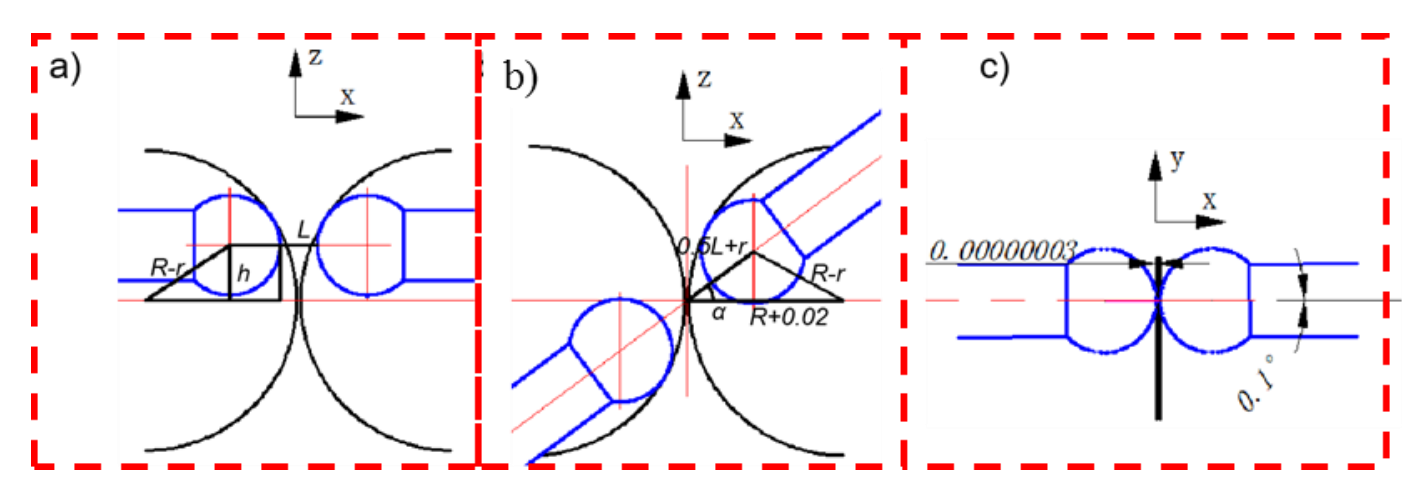

Fig.7 The uncertainty of measurement error due to probe position. a) Error caused by probe eccentricity. b) Measurement error caused by probe deflection in XOZ plane. c) Measurement error caused by probe deflection in XOY plane

It can be seen that the measurement error is not only related to $h$, but also related to the probe radius $r$ and arc radius $R$. Therefore, it is necessary to analyze the effects of $r$ and $R$ on the measurement error separately and determine the specific values of $r$ or $R$, then analyze the impact of the offset $h$ on the measurement error.

Because the radius $\mathrm{r}$ of the probe is $0.5 \mathrm{~mm}$ and the roundness is $0.001 \mathrm{~mm}$. According to Equation(1), we suppose $R=1.5 \mathrm{~mm}, h=5 \mu \mathrm{m}$, and finally get the relationship between $\Delta L$ and $r$, as shown in Fig.8a.

It can be seen from Fig.8a that the measurement error increases with the increase of the probe radius $r$. So we chose the upper limit of $r$, which is $0.501 \mathrm{~mm}$ to analyze the measurement error caused by probe eccentricity.

Suppose $r=0.501 \mathrm{~mm}, h=5 \mu \mathrm{m}$ the range of $R$ is $1.3-1.5 \mathrm{~mm}$, and we get the relationship between $\Delta L$ and $R$. It can be seen from Fig.8b that as $R$ increases, the measurement error of the narrow neck thickness decreases. However, the measurement error decreases in $10^{-3} \mu \mathrm{m}$ scale. Since the actual measurement is 
on-machine detection, the machine tool trims the narrow neck according to the measurement results of the narrow neck radius. Therefore, the measurement error reduces during processing. Consider the two factors above, the influence of $R$ on the measurement error can be ignored. So we take $R=1.5 \mathrm{~mm}$.

taking $r=0.501 \mathrm{~mm}$ and $R=1.5 \mathrm{~mm}$ and it obtain the curve of $\Delta L$ against $h$, as shown in Fig.8c:
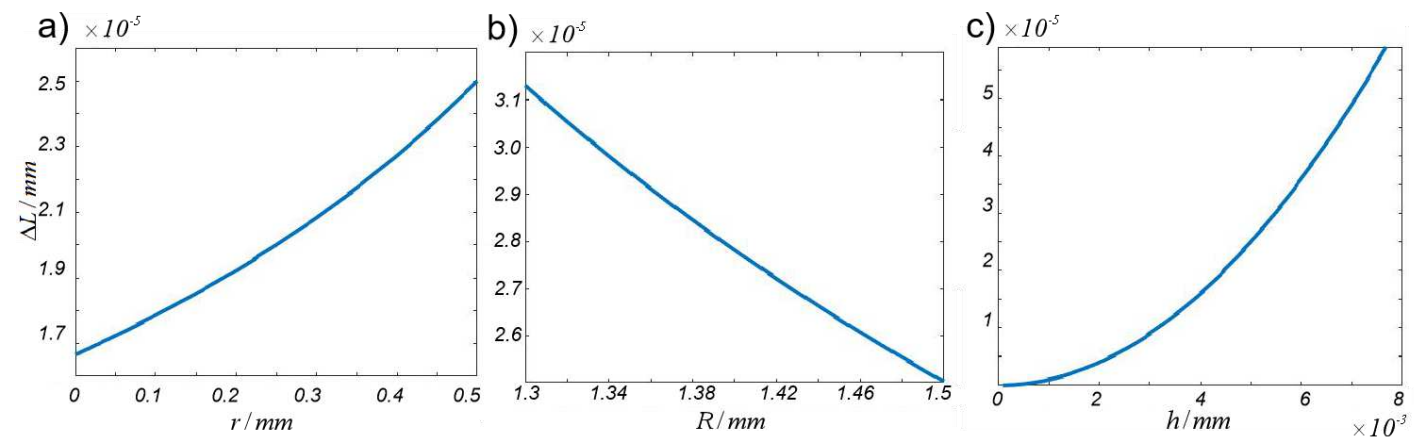

Fig.8 Curve of measurement error. a) Curve of measurement error against $r$. $\boldsymbol{b}$ ) Curve of measurement error against $R$. c) Curve of measurement error $\Delta L$ against $h$

The linear motion error of our machine tool is $5 \mu \mathrm{m}$, From Fig.8c, the error caused by the eccentricity of the probe is approximately $0.025 \mu \mathrm{m}$.

Due to the rotation error of the B-axis of the machine tool, the probe may deviate around the $y$-axis after being fixed. The schematic diagram of measurement when the probe is deflected in $\mathrm{XOZ}$ plane is shown in Fig.7b:

The rotation accuracy of the $\mathrm{B}$ axis can reach $0.001^{\circ}$,i.e. $\alpha=0.001^{\circ}$,from the law of cosines, we can obtain Equation(2),

Take $r=0.5 \mathrm{~mm}$ and $R=1.5 \mathrm{~mm}$. Obviously, the measuring error caused by the probe deflection in $\mathrm{XOZ}$ plane increases as the deflection angle increases. Take the maximum deflection angle $\alpha=0.001^{\circ}$, according to Equation(3), the error calculated is $\Delta L=2.39 \times 10^{-10} \mathrm{~mm}$, which can be ignored.

$$
\begin{gathered}
(R-r)^{2}=(R+0.02)^{2}+(0.5 L+r)^{2}-2(0.5 L+r)(R+0.02) \cos (a) \\
\Delta L=2 L-2 r-0.04
\end{gathered}
$$

Due to the manufacturing error of the device, the longitudinal direction of V-groove and the base can not be guaranteed to be strictly parallel, so the probe will have a certain deflection around the $\mathrm{Z}$ axis. If the $\mathrm{V}$-groove of the base is processed according to IT6 standard, the reference length of the measuring device is $150 \mathrm{~mm}$. 
According to the mechanical design manual, the parallelism requirement is $30 \mu m$. Then the parallelism between the sensor probe and the bottom surface is required to be $30 \mu \mathrm{m}$. So the maximum angle between the probe line and the base is arctan $(0.03 / 150)=0.011^{\circ}$.

In $\mathrm{CAD}$, the central line of the probe deflects $0.1^{\circ}$ from the $\mathrm{Z}$ axis, and we use CAD to measure the error caused by the deflection(Fig.7c). It is found that the unilateral error is only $3 \times 10^{-5} \mu \mathrm{m}$ and the total measurement error is $6 \times 10^{-5} \mu \mathrm{m}$, so when the angle between the probe axis and the base is $0.011^{\circ}$, the error will be greatly reduced. And here, the error can be ignored.

\section{(2) The measurement error due to temperature change}

The temperature drift given by the instruction is $0.15 \mu \mathrm{m} /{ }^{\circ} \mathrm{C}$. Suppose that the temperature range is $20 \pm 0.5^{\circ} \mathrm{C}$, the error due to temperature change is $0.15 \mu \mathrm{m}$. The material of the flexible joint is $3 J 33$ constant elastic alloy steel. The thermal expansion coefficient is $1.8 \times 10^{-5} /{ }^{\circ} \mathrm{C}$ under the condition that the room temperature is $20 \pm 0.5^{\circ} \mathrm{C}$, the measurement error caused by the deformation of workpiece when temperature change is $0.00072 \mu \mathrm{m}$, which can be ignored.

\section{(3)The measurement error due to measuring force}

The measuring force provided by the instruction is $0.63 \mathrm{~N}$. We simulated the deformation of the narrow neck during the measuring process. The simulation result is shown in Fig.9, we found the maximum displacement is $0.02 \mu m$.

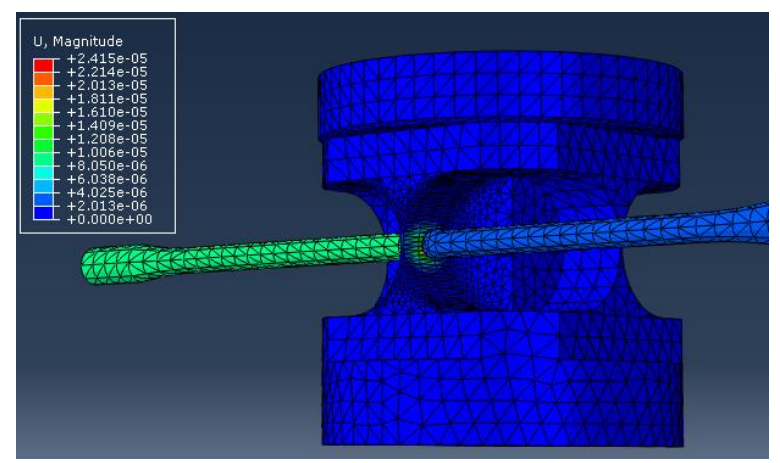

Fig.9 The deformation of the narrow neck

(4) The measurement error due to the sensor performance

As it mentioned above, the linearity of the sensor is $0.07+0.4 L(\mu m)$, which is the average linearity in the full range of the sensor. In actual measurement, we use 
calibrated gauge blocks as the standard. Therefore, the number on TT80 is actually the deviation between the gauge and narrow neck thicknesses. This deviation is usually very small, which is less than $5 \mu m$ in actual measurement. Therefore, the range $\pm 5 \mu m$ can be used, in which we can make full use of the high linearity in small range. From the test conducted by professional measuring institutions, the linearity of the sensor in $\pm 5 \mu m$ range is not more than $0.01 \mu m$, the repeatability of the sensor probe is not more than $0.015 \mu \mathrm{m}$. Since two sensors are used, the linearity and the repeatability needs to be multiplied.

Considering the error sources above, we assume that each error input is independent each other,total error is not more than $0.25 \mu \mathrm{m}$.

The contribution of each error source to the overall measurement uncertainty is plotted as a histogram. As shown in the Fig.10, the error caused by temperature drift accounts for $60 \%$ of the total error component approximately, followed by the sensor's repeatability, linearity, and the eccentricity of probes. Since the object is a narrow-walled structure, the deformation of the workpiece caused by the measuring force cannot be ignored. And because the motion accuracy of the machine tool and the characteristics of the sensor have been determined, it is necessary to reduce the temperature fluctuation as much as possible to improve measurement accuracy. In addition, the uncertainty caused by device manufacturing error is small and can be ignored.

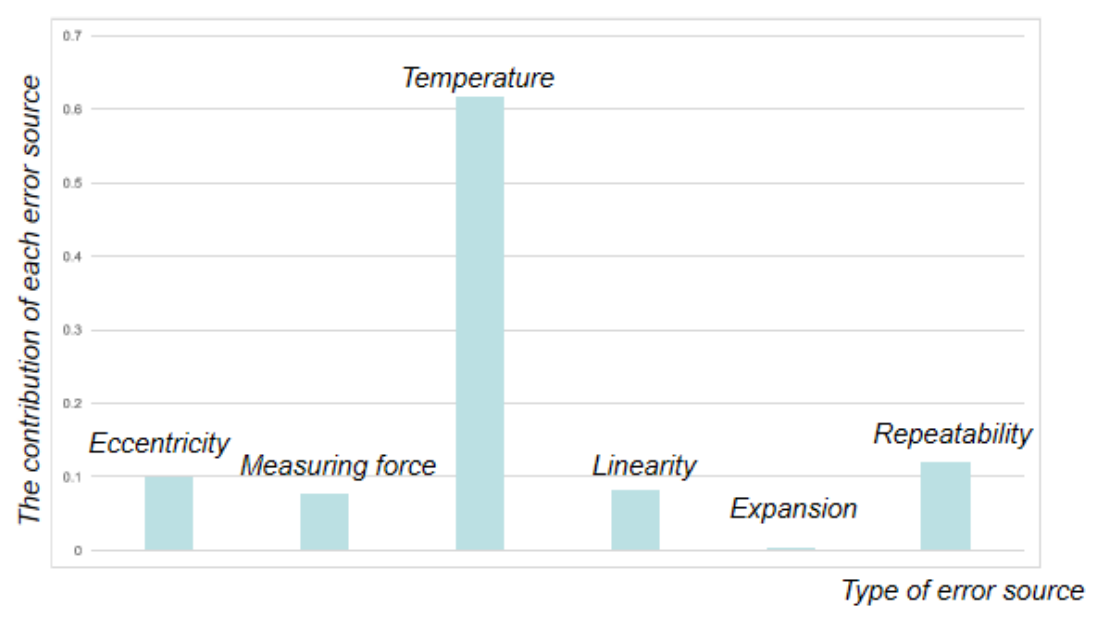

Fig.10 The contribution of each error source 


\subsection{Uncertainty analysis}

Uncertainty analysis is necessary for it indicates the degree to which the measured value cannot be determined due to the existence of measurement error.

According to the uniform probability density distribution, the motion error uncertainty of our machine tool is $5.77 \mu \mathrm{m}$, the error is $0.028 \mu \mathrm{m}$ according to Fig.8c. So the error uncertainty of the probe caused by the eccentricity is described by Equation(4).

$$
\mu_{\mathrm{e}}=\frac{0.028}{\sqrt{3}}=0.016 \mu \mathrm{m}
$$

Samely,the uncertainty of temperature change is $0.577^{\circ} \mathrm{C}$, so the measurement error uncertainty caused by the temperature is given by the Equation(5).

According to 3.2 , the measurement error uncertainty caused by the deformation of workpiece when temperature change is formulated as Equation(6).

$$
\begin{aligned}
& u_{\text {temp }}=\frac{0.15 \times 0.577}{\sqrt{3}}=0.05 \mu \mathrm{m} \\
& u_{d}=\frac{40 \times 1.8 \times 10^{-5}}{\sqrt{3}}=0.00042 \mu \mathrm{m}
\end{aligned}
$$

Take the confidence interval as $95 \%$, the expanded uncertainty coefficient $K=2$, the total expanded uncertainty can be obtained as Equation(7).

$$
\mu=K \times \sqrt{\mu_{\mathrm{p}}^{2}+\mu_{\mathrm{tem}}^{2}+\mu_{\mathrm{s}}^{2}+\mu_{\mathrm{temx}}^{2}+\mu_{\mathrm{c}}^{2}+\mu_{F}^{2}}=0.1 \mu \mathrm{m}
$$

\section{Conclusions}

This present work proposed a measurement method based on the comparative measurement method and the on-machine measurement technology to achieve high-precision and efficient cross-scale narrow neck thickness measurement of flexible joints, and the detailed measurement error analysis was performed.

(1) The comparative measurement method by using a standard gauge block as zero reference was proposed based on the high linearity characteristic in the small measuring range and with the inner calculation mode ' $A+B$ ' of the sensor, achieving 
the high-precision cross-scale measurement of narrow neck thickness of flexible joints.

(2) The on-machine measurement device integrated with an ultra-precision machine tool was developed. The workpiece is clamped on the spindle to complete one-time processing, which eliminates the processing error caused by multiple clamping in traditional processing and improved measurement efficiency. The measuring device was calibrated under static standard conditions by using a digital display horizontal length measuring instrument as standard, and the linearity of the measuring device is $0.25 \%$.

(3) Experimental results showed that the measurement error of the measurement method proposed in this paper is less than $0.15 \mu \mathrm{m}$, and the repeatability is less than $0.1 \mu \mathrm{m}$, which proves the effectiveness of the method. It can also provide technical supports for the high-precision and high-quality measuring process of flexible micro-functional parts.

(4) The theoretical measurement error and uncertainty caused by machine tool motion, manufacturing, measuring force, environmental factor and sensors' characteristics has been analyzed. The environmental factor only include temperature change and exclude factors like brightness, humidity and so on. The measurement error caused by the temperature is more significant while the one caused by the device manufacturing error can be ignored. So the total error can be reduced by building a constant temperature room or increasing thermal inertia, such as expanding the machining area.

\section{Acknowledgements}

This work is supported by Beijing Institute of Technology Research Fund Program for Young Scholars (Grant No. 2020CX04060) and China Postdoctoral Science Foundation (Grant No. 2020M670152).

\section{Declaration}

Ehical approval: All the authors declare that no animals or human participants are involved in this research. There are no ethical issues to declare. 
Consent to participate: All the authors declare that no human participants are involved in this research. Only authors participate in the research work of this paper.

Consent to publish: All authors agree to publish the research results in this paper.

Authors contributions: X.Jin: Conceptualization, Data curation, Validation. R.L. Gao: Writing -original draft, Data curation. C.J. Li: Formal analysis, Funding acquisition, Methodology, Project administration. Z.P. Zheng: Investigation, Visualization. M.Z. Xiao and Z. Zuo: Writing -reviewing \& editing, Funding acquisition.

Funding: This research is supported by Beijing Institute of Technology Research Fund Program for Young Scholars (Grant No. 2020CX04060) and China Postdoctoral Science Foundation (Grant No. 2020M670152).

Competing interests: We declare that we have no financial and personal relationships with other people or organizations that can inappropriately influence our work. This manuscript has not been published or presented elsewhere in part or in entirety and is not under consideration by another journal. I have read and understood your journal's policies, and I believe that neither the manuscript nor the study violates any of these.

Availability of data and materials: The datasets used or analysed during the current study are available from the corresponding author or the first author on reasonable request.

\section{References}

[ 1 ] Schneider,F.,Das,J.,Kirsch,B.et al.(2019)Sustainability in Ultra Precision and Micro Mach-ining: A Review. International Journal of Precision Engineering and Manufacturing-Green Technology.6(3), 601-610.https://doi.org/10.1007/s40684-019-00035-2

[2] Yu D P , Gan S W , Yoke San Wong.(2012)Optimized tool path generation for fast tool servo diamond turning of micro-structured surfaces[J]. International Journal of Advanced Manufacturing Technology, 63(9-12):1137-1152.https://doi.org/10.1007/s00170-012-3964-z

[3] Sazedur Rahman, M., Saleh, T., Lim, H. S., Son, S. M., \& Rahman, M. (2008). Deve lopment of an on-machine profile measurement system in ELID grinding for machining as pheric surface with software compensation. International Journal of Machine Tools and Ma nufacture,48(7), 887-895.https://doi.org/10.1016/j.ijmachtools.2007.11.005

[4] Zou X , Zhao X , Li G , et al.(2017)Non-contact on-machine measurement using a chromatic confocal probe for an ultra-precision turning machine. The International Journal of Advanced Manufacturing Technology. 90(5-8):1-10.https://doi.org/10.1007/s00170-016-9494-3

[5] Zhao J,Sang Y,Duan F,et al(2019)Improvement of measurement resolution of a mechan ical touch-trigger probe method for static radial runout measurements based on digital ima ge correlation.The International Journal of Advanced Manufacturing Technology.105(7-8):31 27-3136.https://doi.org/10.1007/s00170-019-04557-6

[6] Lian M,Liu H,ZhangT,et al(2019)Ultrasonic on-machine scanning for thickness measure ment of narrow-walled parts:modeling and experiments.The International Journal of Advanc ed Manufacturing Technology.104:2061-2072.https://doi.org/10.1007/s00170-019-04021-5

[7] H.H.Shahabi,M.M.Ratnam(2010)Noncontact roughness measurement of turned parts usin $\mathrm{g}$ machine vision.The International Journal of Advanced Manufacturing Technology.46:275284.https://doi.org/10.1007s00170-009-2101-0

[8] Böhm J,Jech M,Vellekoop M.(2010)Analysis of NM-Scale Scratches on High-Gloss Tri 
-bological Surfaces by Using an Angle-Resolved Light Scattering Method. Tribology Letter s.37(2):209-214.https://doi.org/10.1007/s11249-009-9517-4

[9] Beckwith,TG,and RD Marangoni(1990)Mechanical Measurements. Addison Wesley,New J-ersey.

[10] Yan,G.,You,K.\& Fang,F.(2020).Three-Linear-Axis Grinding of Small Aperture Aspheric Surfaces.International Journal of Precision Engineering and Manufacturing-Green Technology. 7(7), 997-1008 https://doi.org/10.1007/s40684-019-00103-7

[11] Suzuki H,Onishi T,Moriwaki T,Fukuta M,Sugawara J(2008)Development of a $45^{\circ}$ tilte don-machine measuring system for small optical parts. CIRP Annals Manufacturing Techno logy.57(1):411-414.https://doi.org/10.1016/j.cirp.2008.03.041

[12] Chen F,Yin S,Huang H,Ohmori H,Wang Y,Fan Y,Zhu Y(2010)Profile error compensat i-on in ultra-precision grinding of aspheric surfaces with on-machine measurement. Internat ional Journal of Machine Tools Manufacture 50(5):480-486.https://doi.org/10.1016/j.ijmachto ols.2010.01.001

[13] Zhang X,Zeng Z,Liu X,Fang F(2015)Compensation strategy for machining optical free -form surfaces by the combined on and off-machine measurement. Optics express 23(19):2 4800-24810.https://doi.org/10.1364/OE.23.024800

[14]Noh YJ,Arai Y,Gao W(2009)Improvement of a fast tool control unit for cutting force measurement in diamond turning of microlens array. International Journal of Surface Scien ce and Engineering 3(3):227-241.https://doi.org/10.1504/IJSURFSE.2009.026611

[15] Lee K,Noh Y,Arai Y,Shimizu Y,Gao W(2011)Precision measurement of microlens pro fi-le by using a force-controlled diamond cutting tool on an ultra-precision lathe. Internatio nal Journal of Precision Technology2(2/3):211-225.https://doi.org/10.1504/IJPTECH.2011.039 460

[16] Li D,Tong Z,Jiang X,Blunt L,Gao F(2018)Calibration of an interferometric on-machine probing system on an ultra-precision turning machine.Measurement.118:96-104.https://doi.org /10.1016/j.measurement.2017.12.038

[17] Li D,Cheung CF,Ren M,Whitehouse D,Zhao X(2015)Disparity pattern-based autostereo -scopic 3D metrology system for in situ measurement of microstructured surfaces. Optics Letters 40(22):5271-5274.https://doi.org/10.1364/ol.40.005271

[18] Shibuya A, Arai Y, Yoshikawa Y, et al.(2010)A spiral scanning probe systemfor micr o-aspheric surface profile measurement. International Journal of Advanced Manufacturing $T$ echnology,46(9):845-862.https://doi.org/10.1007/s00170-008-1812-y.

[19] Santoso, T., Syam, W.P., Darukumalli, S. et al.(2020) On-machine focus varia-tion me asurement for micro-scale hybrid surface texture machining.The International Journal of Ad vanced Manufacturing Technology 109, 2353-2364.https://doi.org/10.1007/s00170-020-05767-z [20] Croft WJ(2006)A Brief History of Microscopy.World Scientific Publishing Co-mpany, Singapore.

[21] Li D,Wang B,Tong Z,et al.(2019)On-machine surface measurement and applic-ations $\mathrm{f}$ or ultra-precision machining:a state-of-the-art review.The International Journal of Advanced Manufacturing Technology,104(1-4).https://doi.org/10.1007/s00170-019-03977-8.

[22] Hecht E.(2002)Optics.4th,Addison Wesley,New Jersey.

[23] Zhao, Z., Ding, D., Fu, Y.et al.(2019)A hybrid approach for measurement th-ickness of complex structural parts using ultrasonic inspection and on-machine p-robing.The Interna tional Journal of Advanced Manufacturing Technology.103, 4777-4785. https://doi.org/10.100 7/s00170-019-04025-1.

[24] Avraham Lorber,Bruce R,Kowalski.(1988)Estimation of prediction Error for Multivariat -e Calibration. Journal of Chemometrics.2(2):93 109,https://doi.org/10.1002/cem.1180020203.

[25] Shin, DYYoo, SS.,et al. (2015)Uncertainty analysis in contact resistivity measurements of crystalline silicon solar cells. International Journal of Precision Engineering and Manufa cturing Green Technology 2(3),237-244.https://doi.org/10.1007/s40684-015-0028-5 
Figures

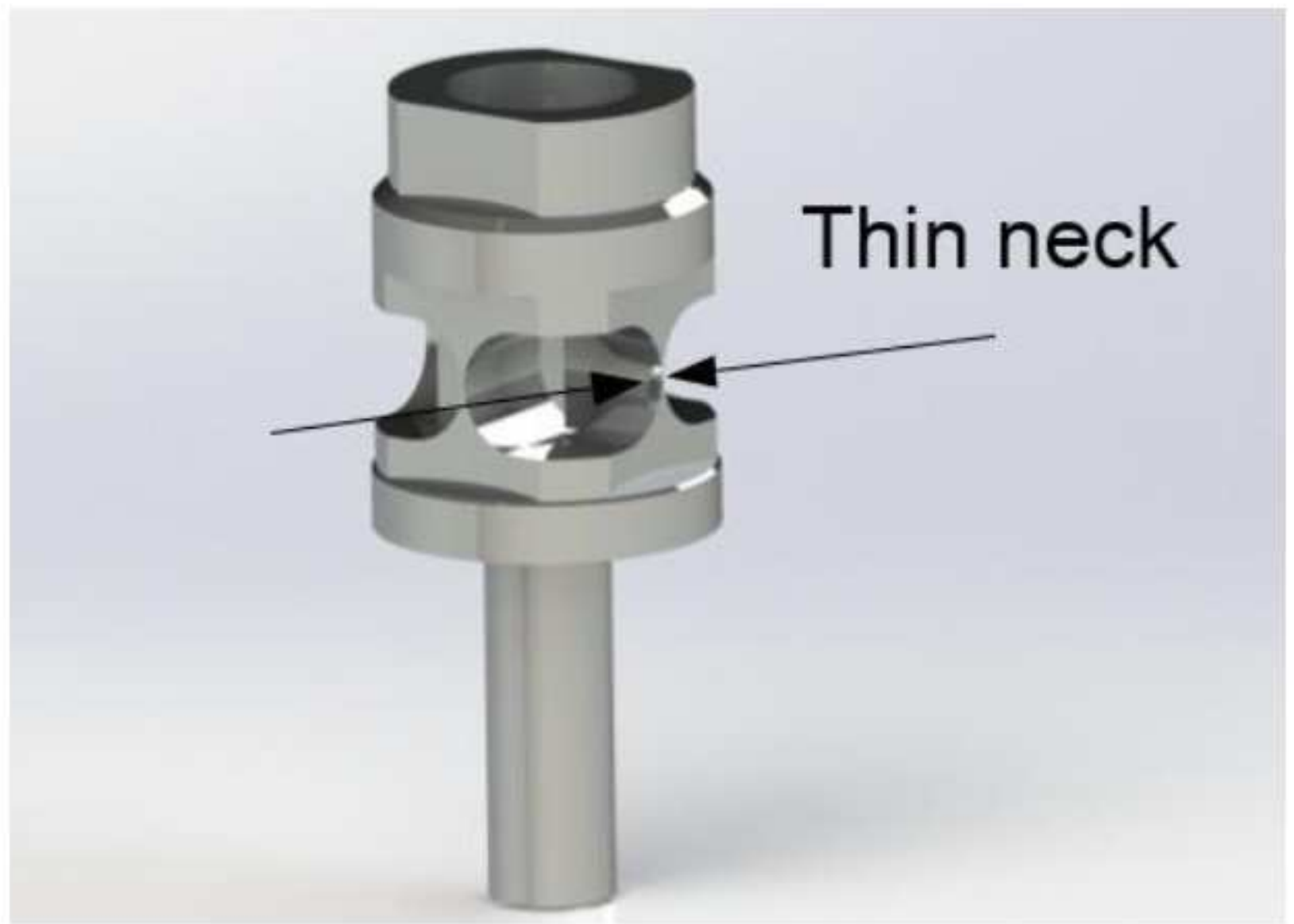

Figure 1

The flexible joint
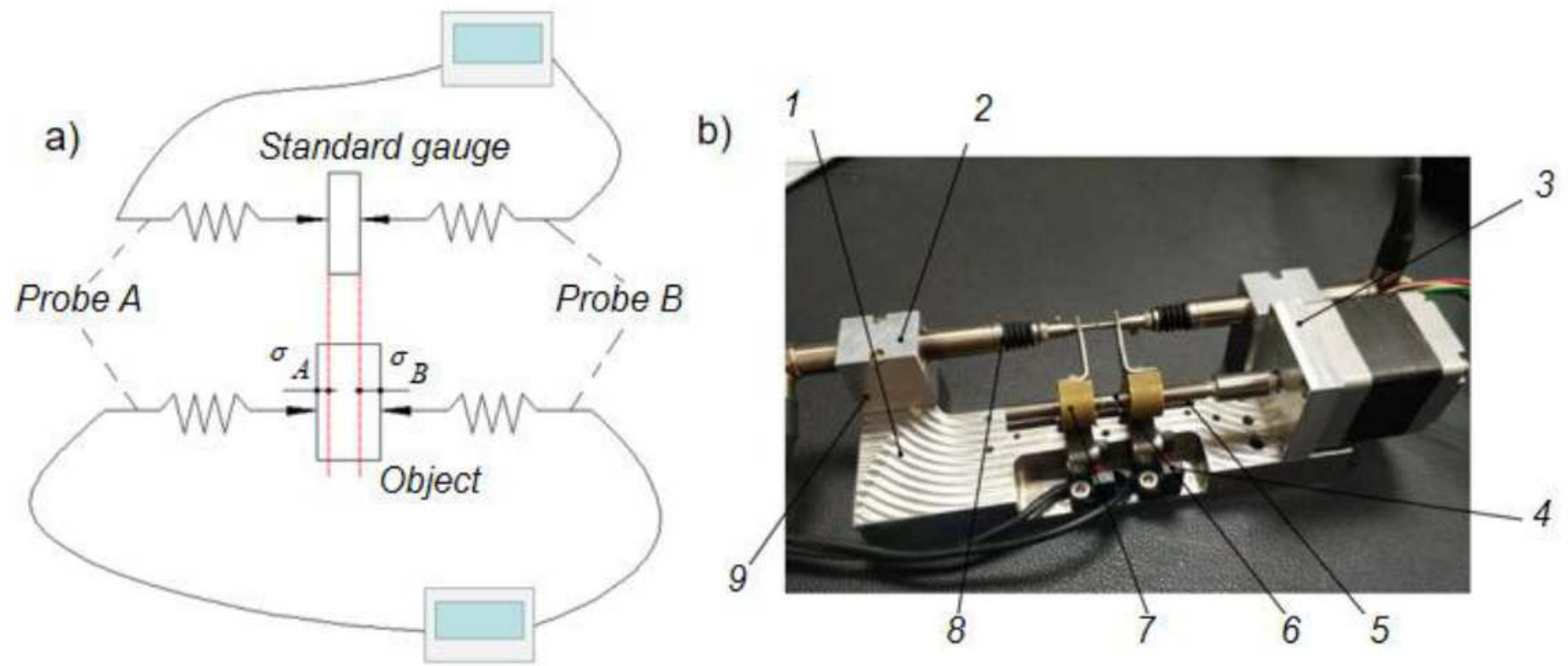

Figure 2 
Measuring method. a) comparative measurement principle. b) measuring device.

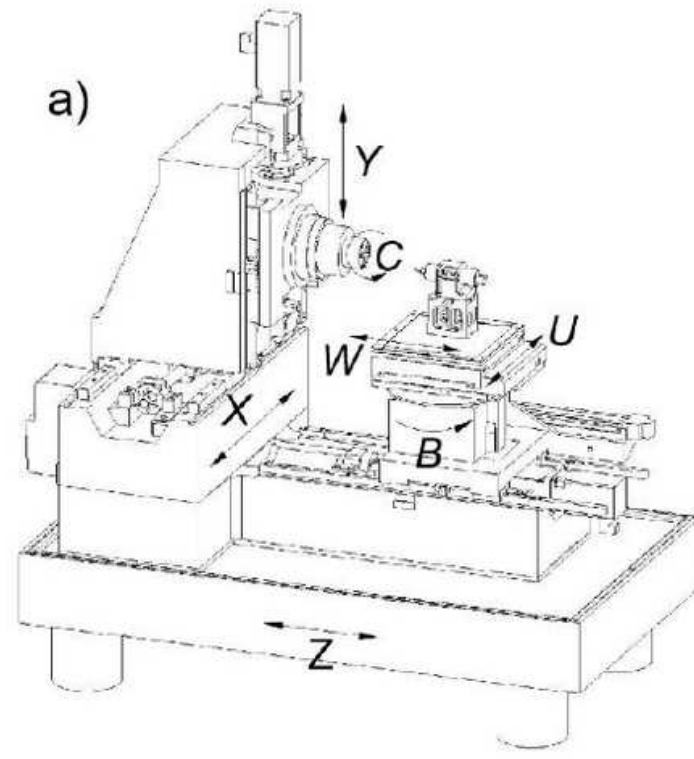

b)

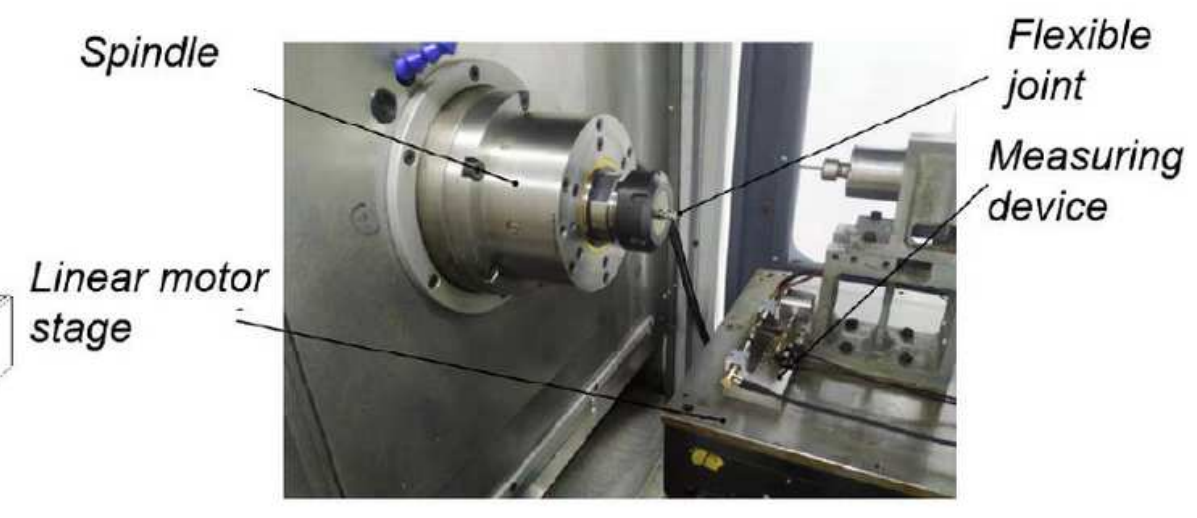

Figure 3

The carrier for the on-machine measurement. a) Machine coordinate system. b) Position of the measuring device

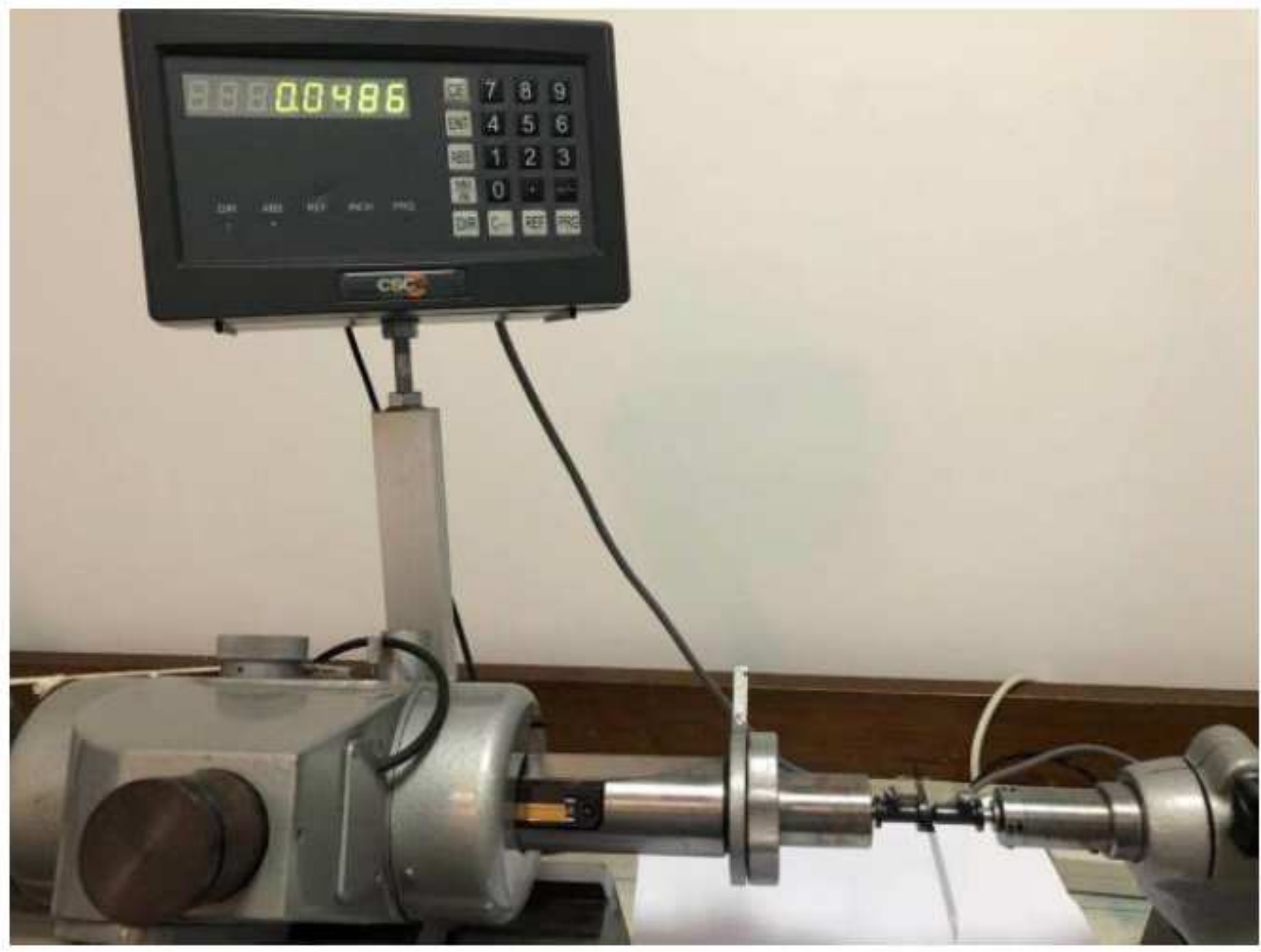


Figure 4

A digital display horizontal Length measuring instriunent

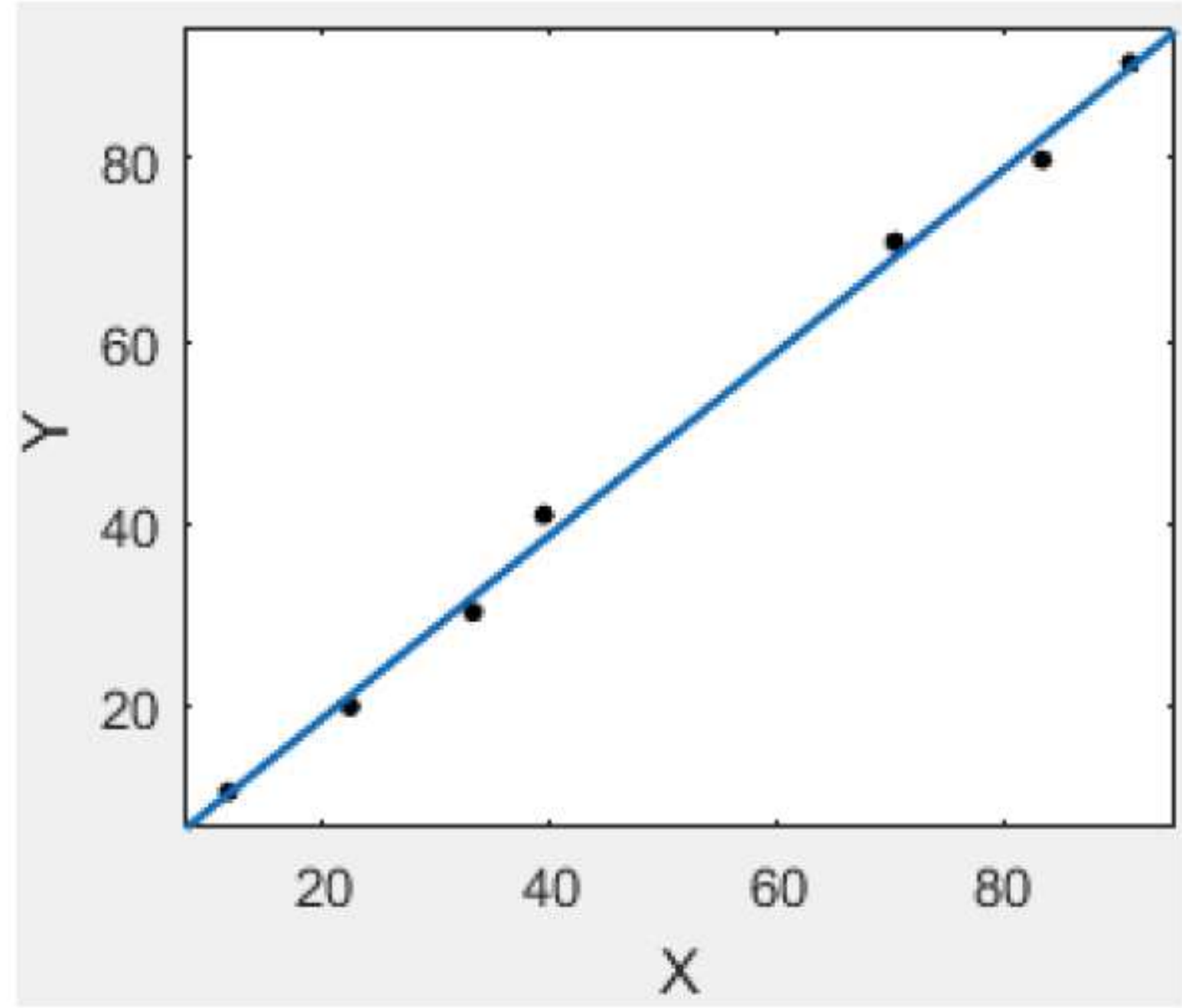

Figure 5

Fitting line

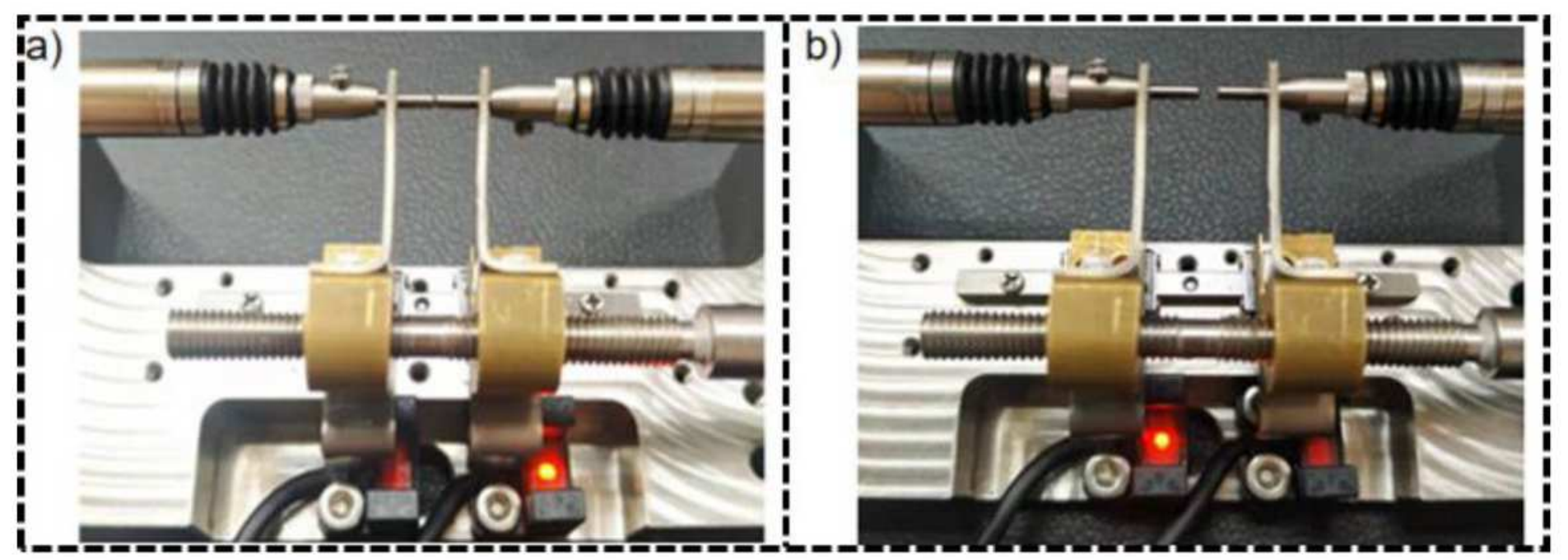


Figure 6

Motion of the probes. a) Two probes contact with each other. b) Two probes move away

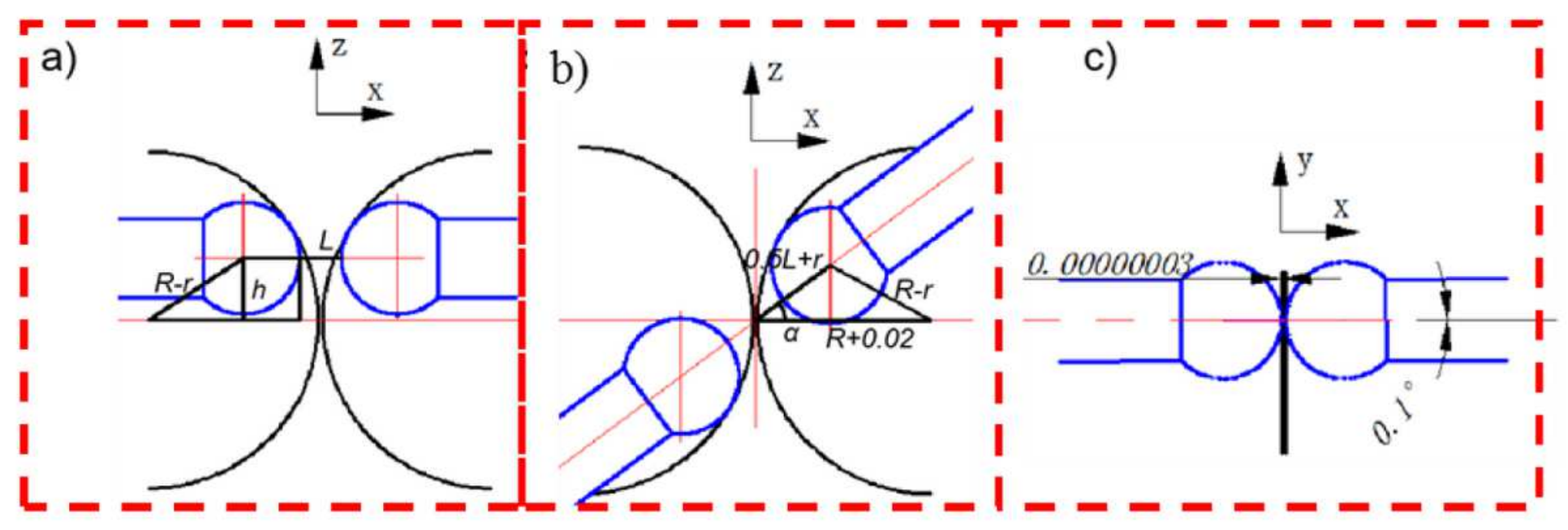

Figure 7

The uncertainty of measurement error due to probe position. a) Error caused by probe eccentricity. b) Measurement error caused by probe deflection in XOZ plane. c) Measurement error caused by probe deflection in XOY plane
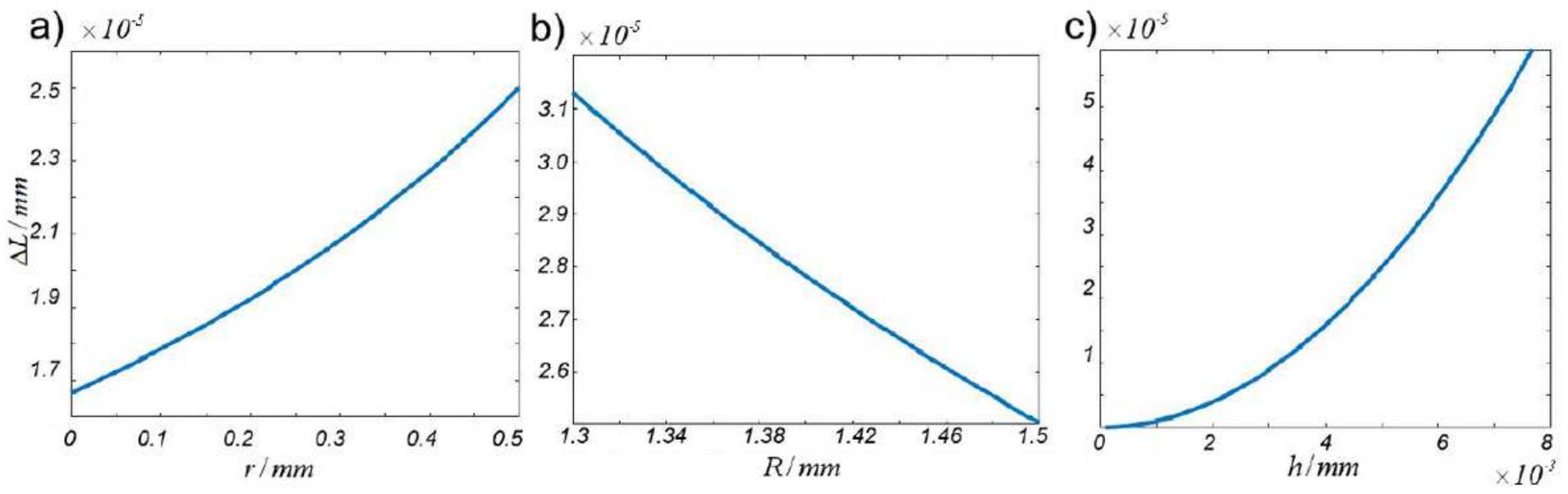

Figure 8

Curve of measurement error. a) Curve of measurement error against $r$ b) Curve of measurement error against R. c) Curve of measurement error $\Delta \mathrm{L}$ against $\mathrm{h}$ 


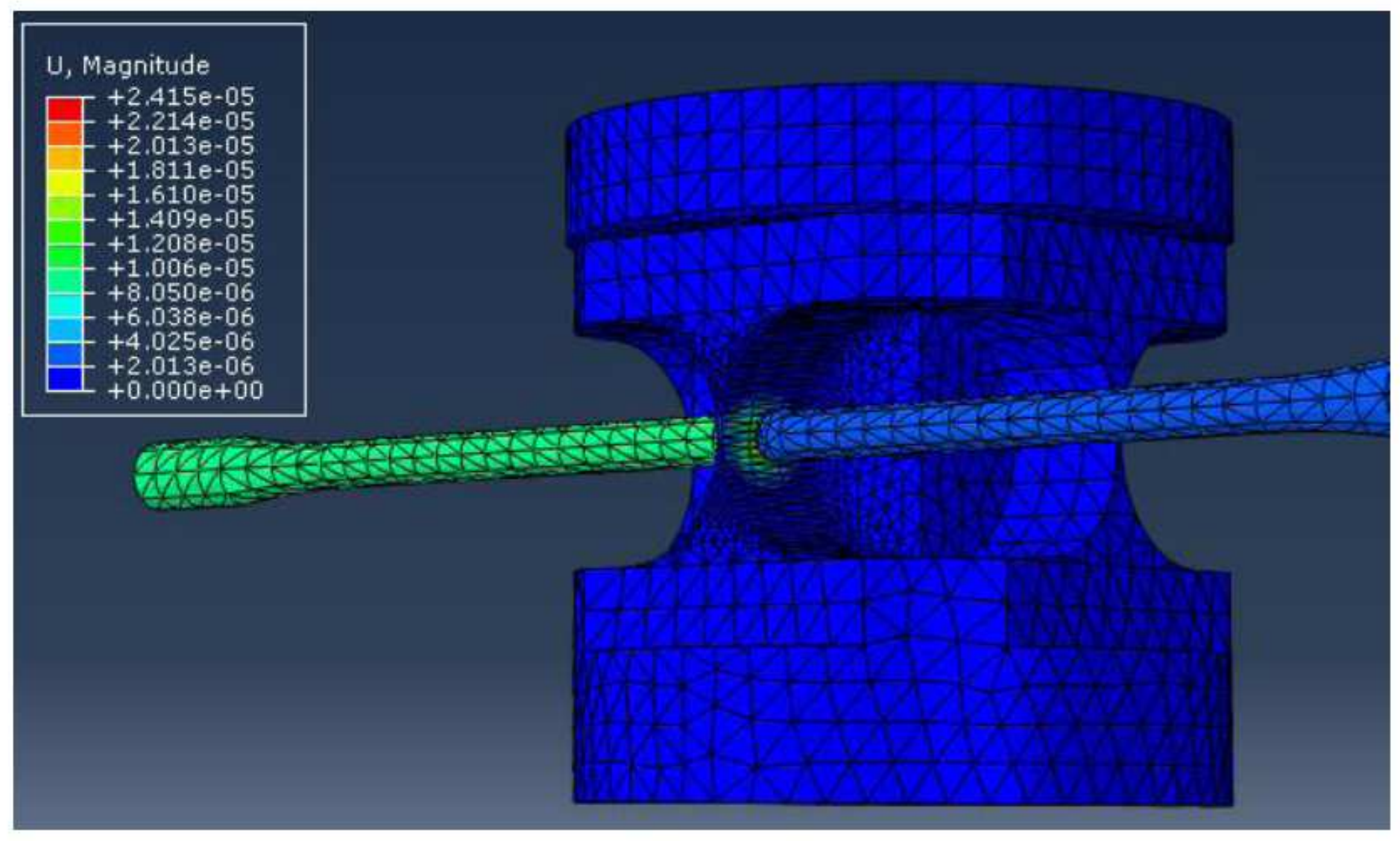

Figure 9

The deformation of the narrow neck

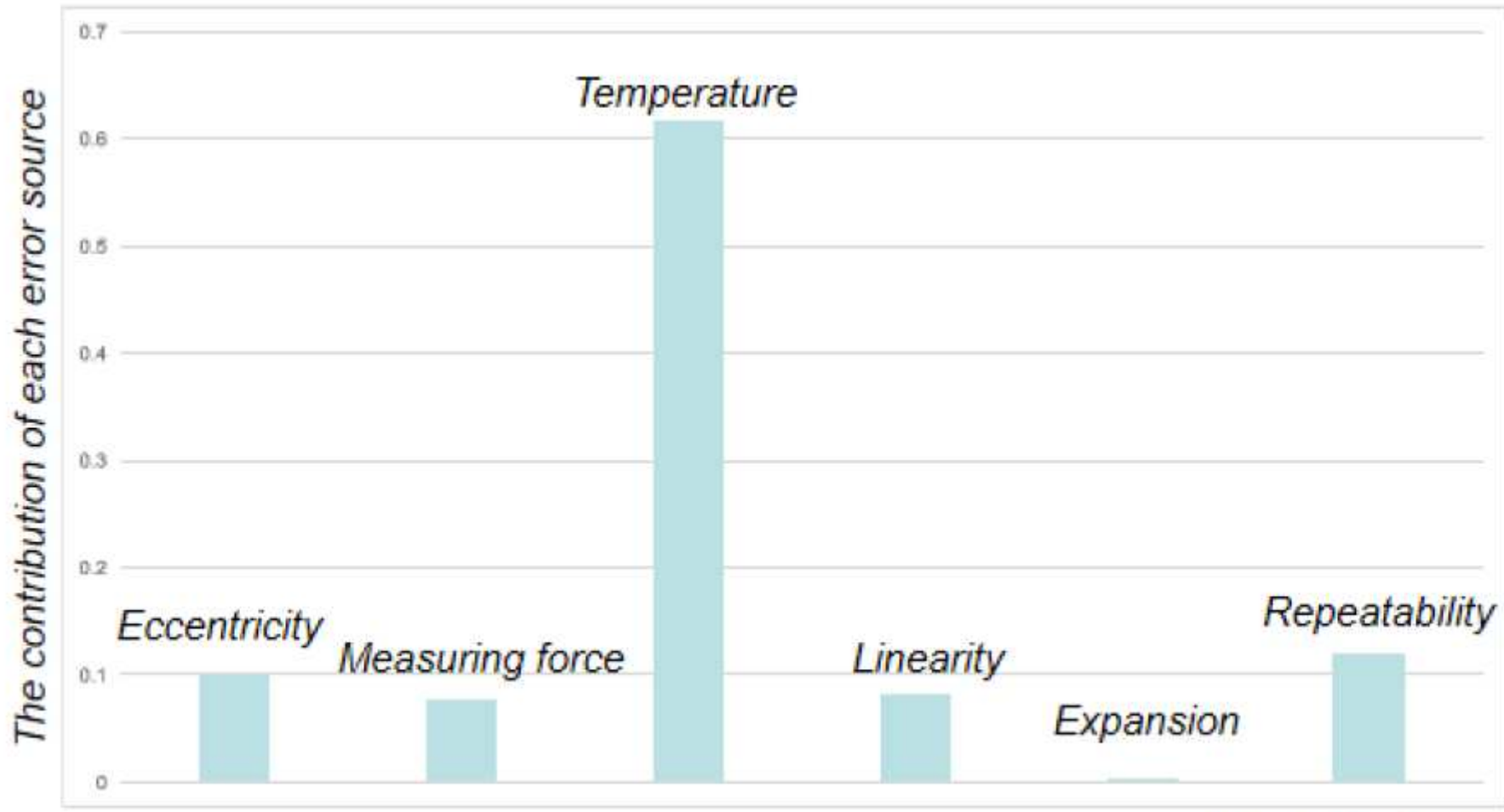


Figure 10

The contribution of each error source 